University of Nebraska - Lincoln

DigitalCommons@University of Nebraska - Lincoln

Faculty Publications, Department of Psychology

Psychology, Department of

May 2005

\title{
Novelty reward as a measure of anhedonia
}

Rick A. Bevins

University of Nebraska-Lincoln, rbevins1@unl.edu

Joyce Besheer

University of North Carolina-Chapel Hill

Follow this and additional works at: https://digitalcommons.unl.edu/psychfacpub

Part of the Psychiatry and Psychology Commons

Bevins, Rick A. and Besheer, Joyce, "Novelty reward as a measure of anhedonia" (2005). Faculty

Publications, Department of Psychology. 42.

https://digitalcommons.unl.edu/psychfacpub/42

This Article is brought to you for free and open access by the Psychology, Department of at DigitalCommons@University of Nebraska - Lincoln. It has been accepted for inclusion in Faculty Publications, Department of Psychology by an authorized administrator of DigitalCommons@University of Nebraska - Lincoln. 


\title{
Novelty reward as a measure of anhedonia
}

\author{
Rick A. Bevins* and Joyce Besheer ${ }^{\S}$
}

\author{
*Department of Psychology, University of Nebraska-Lincoln, Lincoln, NE 68588-0308, USA \\ $\S$ Bowles Center for Alcohol Studies, University of North Carolina-Chapel Hill, Chapel Hill, NC 27599-7178, USA
}

\begin{abstract}
A decrease in sensitivity to pleasurable stimuli, anhedonia, is a major symptom of depression in humans. Several animal models have been developed to simulate this symptom (e.g. drug withdrawal, learned helplessness) using reward-sensitive procedures such as intracranial self-stimulation and progressive ratio responding as a measure of reward function. Recently, we introduced the use of another procedure, novel-object place conditioning in rats, to measure reward function in an associative learning situation. Withdrawal from chronic nicotine blocked a place preference conditioned by access to novel objects. This blockade was not due to impairment of object interaction, general activity, novelty detection, environmental familiarization, or expression of learning. Consequently, nicotine withdrawal directly reduced the rewarding properties of novelty. It is proposed that the novel-object place conditioning procedure could be usefully extended to other experimental situations and to genetically altered mice, so as to better understand the processes underlying changes in reward function.
\end{abstract}

Keywords: Depression; Drug withdrawal; Pavlovian conditioning; Conditioned place preference; Nicotine; Object recognition; Reward learning

\section{Introduction}

According to the World Health Organization depression is one of the primary disabilities that contribute significantly to the individual and fiscal disease burden of most countries. Thus, a better understanding of the etiology of depression could have enormous impact at the individual and societal level. Of the many factors intrinsic to this disorder, the American Psychiatric Association and the World Health Organization include attenuation or loss of enjoyment or pleasure in the diagnostic description of major depression (American Psychiatric Association, 1994). Of interest in the present paper is the symptom of anhedonia or decreased sensitivity to pleasurable events.
At least in part, the prevalence and presumed importance of this symptom has lead several investigators to adopt the use of animal procedures that are sensitive to experimental manipulations in reward value. Intracranial self-stimulation (ICSS) is perhaps one of the better studied examples (Markou and Koob, 1992 and Olds and Milner, 1954). Briefly, steady rates of bar-pressing in rats can be maintained if brief electrical stimulation of the posterior lateral hypothalamus follows the pressing behavior. The required level of stimulation to maintain steady performance is determined for each subject. Shifts in the required current are taken as changes in sensitivity of brain reward. Thus, an increase in the level of ICSS current required to maintain bar-pressing is taken as evidence 
of decreased sensitivity to brain reward (i.e. anhedonia). Most notable from the perspective of the present paper is the consistent demonstration that withdrawal from drugs of abuse such as amphetamine and nicotine produce an increase in ICSS levels (Epping-Jordon et al., 1998 and Leith and Barrett, 1976). Drug abstinence in chronic drug users is often associated with depression-like symptoms including diminished enjoyment (American Psychiatric Association, 1994), and the ICSS procedure has been an especially useful animal model for elucidating the processes mediating withdrawal-induced decreases in reward sensitivity.

Another reward-sensitive procedure that has been recently adopted by researchers is responding under a progressive ratio schedule (Hodos, 1961). In this procedure, the response requirement (e.g. bar-pressing) for a set reward (e.g. sweetened condensed milk) is continually increased until the animal stops responding. Typically, the last response requirement that obtained a reward, termed the 'break point,' is used as a measure of reward magnitude or strength. Weaker or less desirable rewards such as highly diluted sweetened condensed milk control lower break points (cf. Hodos, 1961). Barr and Phillips (1999) recently assessed the effects of amphetamine withdrawal in rats on progressive ratio responding for $0.5 \mathrm{ml}$ of $4 \% \mathrm{su}-$ crose. Amphetamine withdrawal decreased the break point suggesting that the reward value of sucrose had been diminished by the withdrawal state - a conclusion consistent with the ICSS procedure using a reinforcer that requires a consummatory response.

Recently, we introduced the use of another procedure, novel-object place conditioning, which allows assessment of the impact of drug withdrawal on conditioned approach behaviors to reward-associated stimuli (Besheer and Bevins, 2003). The present paper will review the research on the novel-object place conditioning procedure, the impact of nicotine withdrawal on the rewarding effects of novelty in this procedure, and the potential for future research extending its usefulness to the general study of anhedonia.

\section{Place conditioning}

The place conditioning procedure has commonly been used to assess an animal's tendency to approach or avoid environmental cues that have been associated with stimuli believed to be affectively important. This task has been especially popular in studying the rewarding and aversive properties of various drugs of abuse (Bardo and Bevins, 2000 and Carr et al., 1989). For example, a rat will typically spend more time in an environment paired with cocaine over another one paired with saline (Cervo and Samanin, 1995). Importantly, the place conditioning procedure has also been used to assess the rewarding properties of non-drug outcomes such as access to social interaction, food, copulatory opportunity, tickling, wheel running, and novelty (Agmo et al., 1995, Bevins and Bardo, 1999, Calcagnetti and Schechter, 1992, Lett et al., 2000 and Panksepp and Burgdorf, 2003). Similar to drug place conditioning, the typical conditioning procedure involves repeatedly pairing a specific environment with the stimulus of interest, for example, food. The animal receives equal confinement to a second distinct environment without food. After several pairings, a postconditioning test is conducted. During this test, the animal is allowed to move freely between the paired and unpaired environments (no food is present). In rats with restricted access to food, or when a highly preferred food item is used, more time is spent in the environment that had been previously paired with access to food (Agmo et al., 1995, Figlewicz et al., 2001 and Papp, 1989). This preference likely reflects a Pavlovian conditioned association between the exteroceptive stimuli that compose the paired environment and the appetitive (rewarding) effects of the food. That is, the paired environment has acquired appetitive value that elicits conditioned approach behaviors (cf. Bardo and Bevins, 2000 and Panksepp et al., 2004).

\section{Novel stimuli \& place conditioning}

Rats display a tendency to spend more time in novel environments (Hughes, 1965) and to interact more with novel objects (Berlyne, 1950 and Bevins et al., 2002). Some researchers have taken this approach tendency to indicate that novel stimuli are rewarding (Bardo et al., 1996 and Pierce et al., 1990), whereas others have suggested that behaviors elicited by novel stimuli simply take more time to perform than behaviors elicited by more familiar stimuli (Bevins and Bardo, 1999). To determine whether novelty could have a rewarding component, Bevins and Bardo (1999) used a place conditioning procedure in which access to novel objects served as the stimulus of interest (see Fig. 1). Rats were placed in the center area of a chamber and given free access to both distinct end compartments to obtain a measure of initial compartment preference. Then, rats were given access to a novel object during repeated confinements to their non-preferred compartment (i.e. conditioning against a preference). They were similarly confined to their preferred compartment without an object. During the post-conditioning test, in the absence of objects, rats displayed an increase in preference for the environment that had been previously paired with novel objects (see bottom panel of Fig. 1). Notably, a control group that received similar confined exposure to the place conditioning chamber and exposure to objects in the home cage did not 


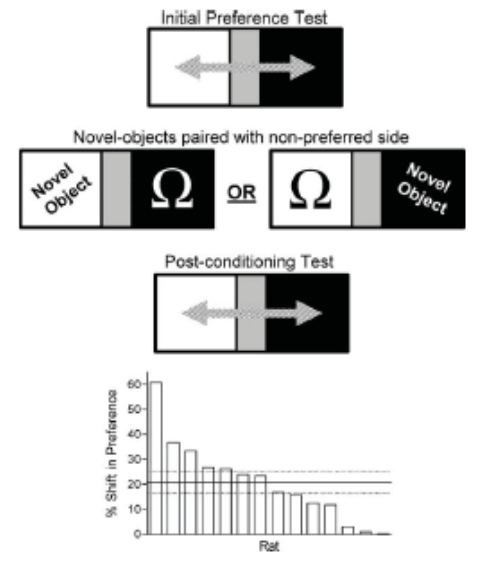

Fig. 1. The upper portion is a graphic representation of a typical novel-object place conditioning protocol using a biased design in which access to novel stimuli are repeatedly paired with a previously determined non-preferred compartment. Similar confined exposure without an object occurs in the non-preferred compartment. The graph on the lower portion of the figure shows the percent shift in preference from the initial preference test to the post-conditioning test for the object-paired compartment for each rat. Solid line represents the mean and the dashed lines denote \pm 1 SEM. Data in graph previously published in a different form (Panksepp et al., 2004).

show a systematic shift in environment preference. This behavioral pattern indicates that access to novel objects, and presumably interacting with those objects, has rewarding properties. These rewarding qualities of novelty become associated with the environment in which they had been repeatedly paired producing an increase in preference for those paired cues (Bevins, 2001).

Subsequent research has shown that novelty of the objects must be maintained in order to condition a preference (Bevins et al., 2002) and that access to novelty can potentiate one-trial intravenous cocaine place conditioning (Bevins, 2001). This latter result suggests a summation or convergence of drug reward and novelty reward. Further, acquisition and expression of novel-object place conditioning involves dopaminergic processes (Bevins et al., 2002 and Besheer et al., 1999) similar to those reported in the place conditioning literature with drugs of abuse. Also, signaled access to novel objects can produce a conditioned increase in activity (Bevins et al., 2002) similar to food and psychomotor stimulants (McFarland and Ettenberg, 1999 and Palmatier et al., 2003). Recently, Dr L.P. Spear's laboratory found that novelty reward as measured in this place conditioning situation varied as a function of sex, age, and housing conditioning (Douglas et al., 2003). For example, singly housed adolescent and adult female rats did not display novelty place conditioning, whereas the group-housed female rats did show a novel-object conditioned place preference. In brief, this work suggests that novelty has re- warding properties that share behavioral and neurobiological processes with other appetitive stimuli and that these rewarding effects might be susceptible to manipulations that alter reward functioning. Of interest in the present paper is the recent adaptation of this novel-object place conditioning procedure to assess altered reward functioning (i.e. anhedonia) during nicotine withdrawal (Besheer and Bevins, 2003).

\section{Novelty reward \& nicotine withdrawal}

Before detailing the modifications made to the novelobject place conditioning procedure and the effects of nicotine withdrawal within these modified procedures, we should briefly discuss the reasons for attempting this avenue of research. The first and perhaps foremost reason was the very clear predictions made by theories of drug withdrawal-induced anhedonia. That is, withdrawal from chronic exposure to a drug such as nicotine should prevent acquisition of an association between environmental cues and the appetitive effects of having access to novelty without interfering with general motor or learning abilities about non-rewards. Indeed, the chronic mild stress model of depression impairs acquisition or expression of place conditioning with food and drugs of abuse in rats (Papp et al., 1991 and Valverde et al., 1997). A second reason, related somewhat to the first, was that generalizing this drugwithdrawal effect to a place conditioning procedure would not only provide multiple converging evidence for observations made in other procedures (e.g. ICSS), but it would open the possibility of studying anhedonia in a Pavlovian-conditioned choice situation. A third reason, more intuitive and speculative on our part, was the seeming face validity of novelty as a rewarding stimulus. In a given day, individuals do not typically experience major rewards such as a job promotion. Rather, the 'everyday' rewards seem to be more individualized and subtle like a conversation with a friend, a dessert after dinner, or noticing more vivid colors in a sunset. To us, at least, it seems like the attenuation or loss of pleasure in the diagnostic description of major depression (American Psychiatric Association, 1994) refers more often to these types of rewards. For rats, we think of access to novelty as one of these more subtle rewards. In support of this idea is our repeated inability to find evidence that access to novelty successfully competes against the presumed bigger reward of a low dose of intravenous cocaine in a place conditioning situation (unpublished data). Further, access to novelty appears rewarding to a majority of control animals. However, there are clear individual differences with a small subset of rats in some experiments showing a decrease rather an increase in preference for the novelty-paired environment (see Fig. 1 and Fig. 2). 


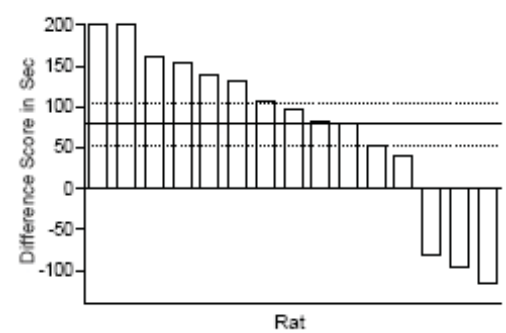

Fig. 2. The difference in time spent between the object paired and unpaired compartments for each rat in a modified one-day novelobject place conditioning procedure that employs an unbiased design. A positive difference score indicates more time in the novelty-paired compartment during the post-conditioning test. Solid line represents the mean and the dashed lines denote \pm 1 SEM. Data previously published in a different form (Besheer and Bevins, 2003)

Whether these individual differences are predictable and related to reward function will have to await further research.

Similar to other place conditioning procedures (see earlier description), the novel-object place conditioning task is a multiple-day procedure. Commonly, the protocol includes an initial preference test or habituation session followed by eight conditioning sessions (e.g. four alternating confinements to each compartment), and lastly a post-conditioning test. Thus, this protocol requires 10 days. In some animal models of depression that include anhedonia as a primary symptom of interest, the decreased reward functioning appears transient. For example, anhedonia as assessed by the ICSS model during nicotine withdrawal lasts 3-4 days (Epping-Jordon et al., 1998 and Harrison et al., 2001). In order to use novel-object place conditioning as a potential associative learning model to assess alterations in reward function that may accompany, for example, drug withdrawal, the standard multiple-day procedures had to be modified.

Ideally, those modifications should result in a oneday procedure so as to be useful for assessing any changes on a daily basis. After several experiments, the effective conditioning protocol was as follows: the initial habituation session was followed by 145 -min conditioning sessions (e.g. seven confinements in each compartment), each confinement was separated by 30 $\mathrm{min}$, and the post-conditioning test occurred $30 \mathrm{~min}$ after the final confinement. Another change from the procedure described earlier was that a non-biased conditioning procedure was used. That is, rather than conditioning against an initial compartment preference, half the rats were randomly selected to receive novel objects paired with one end compartment (e.g. black walls/rod flooring); the remaining rats received novel objects paired with the opposite end compartment (e.g. white walls/mesh flooring). Accordingly, the main dependent measure with this modified procedure was a difference score (time in object-paired com- partment minus time in unpaired compartment) rather than a shift in preference from pre- to post-conditioning. Thus, a positive difference score indicates novelobject conditioning (i.e. novelty reward). Fig. 2 shows the difference scores for 15 rats trained using this oneday novel-object place conditioning procedure. A significant portion of the rats ( 12 of $15 ; \mathrm{p}=0.014$ ), spent more time in the novelty-paired compartment indicating that novelty reward can be assessed with this single-day procedure.

Novelty reward using this model was affected by nicotine withdrawal. Rats undergoing withdrawal from chronic nicotine treatment (i.e. $9 \mathrm{mg} / \mathrm{kg}$ per day hydrogen tartrate delivered for 1 week by an osmotic mini-pump) were tested $1,2,3$, or 4 days after nicotine delivery had been stopped by pump removal (see Besheer and Bevins, 2003 for more detail). As illustrated in Fig. 3, rats did not stay significantly longer in the novelty-paired compartment on days 1,2, and 3 of nicotine withdrawal. However, on day 4 the treated rats, like the controls, again showed preference for the novelty-paired compartment. These findings are consistent with those obtained using the ICSS and progressive ratio models and suggest that withdrawal from nicotine (9 $\mathrm{mg} / \mathrm{kg}$ per day) induces anhedonia that decreases with time. That is, withdrawal-induced decrease in reward sensitivity prevents establishment of an appetitive place preference conditioned by access to novelty.

The blockade of novelty place conditioning was unlikely to be due to anxiety or lack of motivation

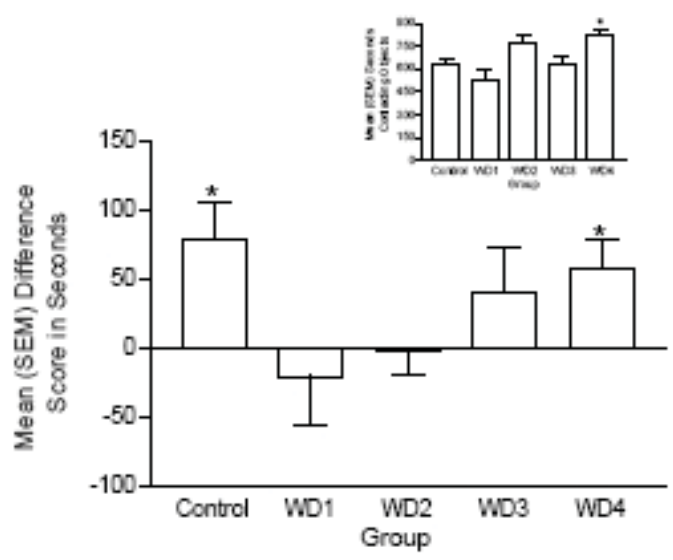

Fig. 3. The mean difference in time spent between the object paired and unpaired compartments in the post-conditioning preference test for controls and rats undergoing withdrawal from chronic nicotine treatment $(9 \mathrm{mg} / \mathrm{kg}$ per day hydrogen tartrate delivered for 1 week by an osmotic mini-pump) 1,2 , 3 , or 4 days after nicotine delivery had been stopped by pump removal (WD1, WD2, WD3, and WD4, respectively). The inset graph denotes mean time spent interacting with objects during the conditioning phase for each group. Data previously published in a different form (Besheer and Bevins, 2003). 
given that nicotine withdrawal did not decrease novel-object interaction (inset graph in Fig. 3). If animals undergoing withdrawal from $9 \mathrm{mg} / \mathrm{kg}$ per day of nicotine hydrogen tartrate had severely diminished interaction with the novel objects, a conditioned association between novelty and the environment might not have developed. Accordingly, loss of the conditioned place preference might be the result of a non-specific motor effect on object interaction rather than a decrease in the affective impact of interacting with novel objects. Notably, the continued approach and interaction with novel objects suggests that withdrawal from nicotine did not induce an anxiogenic response which would be exhibited as an avoidance of the object (see novelty-detection task described later). Further, entries into each compartment during the post-conditioning test (i.e. another measure of motor activity) did not differ among groups indicating that rats readily sampled each end compartment.

The lack of an effect of withdrawal from $9 \mathrm{mg} / \mathrm{kg}$ per day of nicotine on two measures of motor ability, combined with the temporally specific blockade of novelty place conditioning, suggests to us that nicotine withdrawal blunted reward functioning. However, alternative explanations need to be examined. For instance, undergoing withdrawal might have affected the processing of the objects such that the rat was unable to detect that each object was novel during the conditioning sessions. As noted earlier, the novel-object place conditioning procedure relies on the presentation of novel objects; a familiar object does not condition a shift in preference (Bevins et al., 2002). Thus, if the objects were not recognized as novel, place conditioning would not occur. To test this explanation, we used a novel-object detection task that takes advantage of a rat's tendency to interact more with a novel object than a previously experienced sample (familiar) object (Berlyne, 1950). Rats undergoing withdrawal from 9 $\mathrm{mg} / \mathrm{kg}$ per day nicotine hydrogen tartrate during this task readily detected novelty as indexed by more interaction with a novel than a familiar object. Thus, it seems unlikely that loss of place conditioning during withdrawal was due to the rats' inability to detect novelty in the environment. Further, because these rats experienced withdrawal during the object familiarization phase, as well as the novel-object test phase, nicotine withdrawal did not affect processing of information about the sample object (e.g. texture, odor) or the neural storage/use of that information.

The novel-object detection experiment just described indicates that processing of novelty was unaffected. However, that experiment did not assess whether processing of the environment (paired compartment) was impaired by nicotine withdrawal. Such impairment would prevent place conditioning given that a conditioned preference presumably reflects ac- quisition of an association between environmental cues and the appetitive effects of access to novelty. To test this possibility, we took advantage of rats' tendency to interact more with an object if presented in a familiar environment (Bevins et al., 2001 and Sheldon, 1969). Rats that underwent withdrawal from $9 \mathrm{mg} / \mathrm{kg}$ per day of nicotine only during the environmental familiarization phase interacted with a novel object at a level similar to controls that were familiarized with the environment in a non-withdrawal state. If withdrawal impaired familiarization processes, these rats would have shown a decrease in object interaction during the testing phase similar to that of controls never exposed to the environment. In brief, nicotine withdrawal does not affect processes required for learning about environmental cues.

Additionally, follow-up experiments were able to eliminate the possibility that nicotine withdrawal blocked expression of conditioning rather than decreased rewarding properties of novelty during the learning. For example, chronic nicotine-treated rats ( $9 \mathrm{mg} / \mathrm{kg}$ per day hydrogen tartrate) received the oneday novel-object place conditioning protocol previously described. Withdrawal was precipitated with mecamylamine just before the post-conditioning preference test. These rats exhibited novel-object conditioning similar to controls showing that undergoing withdrawal during the test did not interfere with the ability to express the acquired appetitive association. We re-tested this alternative explanation using the novel-object detection task described earlier. In this task, the novel-object discrimination can be interpreted as an 'expression' test. That is, learning about the sample objects in the initial phase of this task is expressed by spending more time interacting with the novel object during testing - detection of novelty requires recall of the familiar. In this study, rats had the sample-object exposure phase while receiving chronic nicotine treatment ( $9 \mathrm{mg} / \mathrm{kg}$ per day); withdrawal was precipitated just before the subsequent novel-object test. Nicotine withdrawal did not affect novel-object detection. Combined, these studies suggest that impairment of novelobject place conditioning was not due to withdrawal interfering with expression of learning.

\section{Future extensions}

Nicotine withdrawal did not impair object interaction, general activity, novelty detection, environmental familiarization, or expression of learning. Elimination of these potential explanations of withdrawal-induced blockade of novelty place conditioning increases our confidence in the original conclusion. That is, the specific blockade of novel-object place conditioning during the early stages of nicotine withdrawal was due to a decrease in the rewarding properties of novelty. This 
conclusion opens the possibility that the novel-object place conditioning task might be an effective tool for assessing changes in reward functioning induced by withdrawal from other abused drugs, and more generally those changes in reward function that occur in other animal models of depression.

On this latter point, the chronic mild stress model of depression was explicitly designed to assess the symptom of anhedonia typically seen in depressed patients (Willner, 1997). In brief, this model exposes the animal to repeated stressors (e.g. wet bedding, new cage mate, etc.) for at least several weeks. Expression of anhedonia in rats exposed to this procedure includes a decreased preference for a dilute sucrose solution, elevated thresholds in the ICSS model, and attenuated place conditioning with appetitive stimuli such as sucrose, amphetamine, and morphine (Papp et al., 1991 and Valverde et al., 1997). This effect on drug and food reward suggests that novelty reward will be readily impacted by chronic mild stress. Notably, the reported duration of depressive symptoms in the chronic mild stress model would permit the use of the multiple-day protocol (cf. Valverde et al., 1997 and Willner, 1997). However, it should be noted that this line of research might be difficult given that that some of the effects of chronic mild stress exposure have not been replicated (Harris et al., 1997 and Hatcher et al., 1997).

Related to this discussion is the learned helplessness model of depression. In this model, the rodent is typically exposed to uncontrollable shock. This exposure results in impairment of escape learning which is taken as a behavioral sign of depression (Greenwood et al., 2003 and Maier and Seligman, 1976). Will animals that developed the behavioral profile described as depressive in this paradigm also show blunted reward functioning as measured by novel-object place conditioning? Careful experimental work will need to eliminate any possibility of generalized fear between the chronic shock situation and the place conditioning apparatus. Conditioned or unconditioned fear behaviors could readily interfere with place conditioning that does not necessarily involve a decrease in reward function.

Regardless of the depression model used to induce anhedonia-e.g. drug withdrawal, chronic mild stress, etc.- the effects of acute and chronic treatment with antidepressant drugs on restoring reward function as measured in the novelty place-conditioning situation will require experimental attention. Further, all the novel-object place conditioning research to date has used rats. Extending this procedure to mice would allow systematic investigation of genetically altered mice that are purported to have depression-like profiles (see Cryan et al., 2002 for a recent review). To do so, will require careful development of a place conditioning protocol that does not include biases that vary across strains (Cunningham et al., 1999; see Cunningham et al., 2003 for a thorough discussion of bias in place conditioning studies). Similar to other functional assays with transgenic mice, any deficit in novelty place conditioning will require detailed empirical work that assesses non-specific alterations in perceptual, motor, and learning abilities before concluding that decreased sensitivity to reward is responsible for blockade.

\section{Concluding thoughts}

We think the potential use of the novel-object place conditioning procedure to assess anhedonia in animal models of depression has exciting possibilities. However, the widespread use of place conditioning might be limited by several factors. First, the nature of the protocol requires the use of between-subject designs. Thus, the number of animals required to complete an experiment can be substantial, especially when an investigator wants to generate a dose-effect function and include all the appropriate controls. Second, and related to the first point, the modified one-day novelty place conditioning is labor intensive. Using two place conditioning chambers, a single replication of rats $(n=8)$ in the experiment described earlier (see Fig. 3) requires an $8.5 \mathrm{~h}$ day with multiple replications needed to complete a single study. Third, the generality of novel-object place conditioning has not been tested beyond Sprague-Dawley rats and three laboratories (M.T. Bardo: University of Kentucky; R.A. Bevins: University of Nebraska-Lincoln; L.P. Spear: Binghamton University). At present, it is unclear whether other rat strains and, as discussed earlier, mice strains will be sensitive to the appetitive effects of novelty. There is no a-priori reason, however, to believe that appropriate parameters in other animals will not be found given that place conditioning with drugs of abuse are readily observed in mice and other rat strains. Finally, to establish that reward function has been decreased, other alternative explanations that can readily affect associative learning must be empirically tested. As detailed earlier, this is an experimentally intensive but necessary step.

In concluding, we would like to make very clear that we are not advocating that place conditioning replace other measures of reward function such as ICSS, progressive ratio responding, sucrose intake, etc. (cf. Forbes et al., 1996). Rather, we are suggesting that novelty place conditioning, or more generally place conditioning with appetitive stimuli such as food, novel objects, or copulatory opportunity, be used in addition to these other measures. A compelling argument for using multiple indices is that it is highly likely that each measure of reward sensitivity is controlled, in part, by different neurobiological and behavioral processes. For instance, at the behavioral level, ICSS in- 
volves sensitivity to a response-outcome relation in which the response option is essentially fixed as the outcome (electrical stimulation) is varied to maintain responding. Although the progressive ratio task also requires maintenance of a response-outcome relation, the response option is varied (increased) as the outcome is held constant. At least intuitively, changes in reward sensitivity as measured by manipulation of different aspects of the response-outcome contingency in these operant conditioning assays is likely controlled by somewhat different functional relations at the behavioral and neurobiological level. In contrast to these operant conditioning procedures, the place conditioning procedure assesses a stimulus-outcome relation (i.e. Pavlovian conditioning). Rats tend to approach appetitive stimuli, thus cues that reliably signal access to these rewarding stimuli (outcomes) tend to control approach or seeking-like behaviors (Bardo and Bevins, 2000 and Panksepp et al., 2004). The place conditioning protocol, thus, assesses anhedonia by determining whether the appetitive association will develop as later expressed in a free-choice situation between a reward associated and a non-reward associated environment. These behavioral and neurobiological processes mediating choice behavior controlled by a stimulus-reward outcome relation will probably differ, at least in part, from those of response-reward outcome relations.

\section{Acknowledgements}

Some of the research described in this report was supported by USPHS grants MH57240, DA11893, and DA06092. We thank K.S. Bevins and M.I. Palmatier for their thoughtful comments on an earlier version of this manuscript.

\section{References}

Agmo et al., 1995 A. Agmo, A. Galvan and B. Talamantes, Reward and reinforcement produced by drinking sucrose: two processes that may depend on different neurotransmitters, Pharmacol. Biochem. Behav. 52 (1995), pp. 403 414.

American Psychiatric Association, 1994 American Psychiatric Association, DSM IV-Diagnostic and Statistical Manual of Mental Disorders (fourth ed.), American Psychiatric Press, Washington, DC (1994).

Bardo and Bevins, 2000 M.T. Bardo and R.A. Bevins, Conditioned place preference: what does it add to our preclinical understanding of drug reward?, Psychopharmacology 153 (2000), pp. 31-43.

Bardo et al., 1996 M.T. Bardo, R.L. Donohew and N.G. Harrington, Psychobiology of novelty seeking and drug seeking behavior, Behav. Brain Res. 77 (1996), pp. 23-43.

Barr and Phillips, 1999 A.M. Barr and A.G. Phillips, Withdrawal following repeated exposure to d-amphetamine decreases responding for a sucrose solution as measured by a progressive ratio schedule of reinforcement, Psychopharmacology 141 (1999), pp. 99-106.
Berlyne, 1950 D.E. Berlyne, Novelty and curiosity as determinants of exploratory behavior, Br. J. Psychol. 41 (1950), pp. 68-80.

Besheer and Bevins, 2003 J. Besheer and R.A. Bevins, The impact of nicotine withdrawal on novelty reward and related behaviors, Behav. Neurosci. 117 (2003), pp. 327-340.

Besheer et al., 1999 J. Besheer, H.C. Jensen and R.A. Bevins, Dopamine antagonism in a novel-object preference and a novel-object place conditioning preparation with rats, $\mathrm{Be}$ hav. Brain Res. 103 (1999), pp. 35-44.

Bevins, 2001 R.A. Bevins, Novelty seeking and reward: implications for the study of high-risk behaviors, Curr. Dir. Psychol. Sci. 10 (2001), pp. 189-193.

Bevins and Bardo, 1999 R.A. Bevins and M.T. Bardo, Conditioned increase in place preference by access to novel objects: antagonism by MK-801, Behav. Brain Res. 99 (1999), pp. 53-60.

Bevins et al., 2001 R.A. Bevins, J. Koznarova and T.J. Armiger, Environmental familiarization in rats: differential effects of acute and chronic nicotine, Neurobiol. Learn. Mem. 75 (2001), pp. 63-76.

Bevins et al., 2002 R.A. Bevins, J. Besheer, M.I. Palmatier, H.C. Jensen, K.S. Pickett and S. Eurek, Novel-object place conditioning: behavioral and dopaminergic processes in expression of novelty reward, Behav. Brain Res. 129 (2002), pp. 41-50.

Calcagnetti and Schechter, 1992 D.J. Calcagnetti and M.D. Schechter, Place conditioning reveals the rewarding aspect of social interaction in juvenile rats, Physiol. Behav. 51 (1992), pp. 667-672.

Carr et al., 1989 G.D. Carr, H.C. Fibiger and A.G. Phillips, Conditioned place preference as a measure of drug reward. In: J.M. Liebman and S.J. Cooper, Editors, The Neuropharmacological Basis of Reward, Clarendon Press, Oxford (1989), pp. 264-319.

Cervo and Samanin, 1995 L. Cervo and R. Samanin, Effects of dopaminergic and glutamatergic receptor antagonists on the acquisition and expression of cocaine conditioning place preference, Brain Res. 673 (1995), pp. 242-250.

Cryan et al., 2002 J.F. Cryan, A. Markou and I. Lucki, Assessing antidepressant activity in rodents: recent developments and future needs, Trends Pharmacol. Sci. 23 (2002), pp. 238-245.

Cunningham et al., 1999 C.L. Cunningham, S.D. Dickinson, N.J. Grahame, D.M. Okorn and C.S. McMullin, Genetic differences in cocaine-induced conditioned place preference in mice depend on conditioning trial duration, Psychopharmacology 146 (1999), pp. 73-80.

Cunningham et al., 2003 C.L. Cunningham, N.K. Ferree and M.A. Howard, Apparatus bias and place conditioning with ethanol in mice, Psychopharmacology 170 (2003), pp. $409-422$.

Douglas et al., 2003 L.A. Douglas, E.I. Varlinskaya and L.P. Spear, Novel-object place conditioning in adolescent and adult male and female rats: effects of social isolation, Physiol. Behav. 80 (2003), pp. 317-325.

Epping-Jordon et al., 1998 M.P. Epping-Jordon, S.S. Watkins, G.F. Koob and A. Markou, Dramatic decreases in brain reward function during nicotine withdrawal, Nature 393 (1998), pp. 76-79.

Figlewicz et al., 2001 D.P. Figlewicz, M.S. Higgins, S.B. NgEvans and P.J. Havel, Leptin reverses sucrose-conditioned place preference in food-restricted rats, Physiol. Behav. 73 (2001), pp. 229-234.

Forbes et al., 1996 N.F. Forbes, C.A. Stewart, K. Matthews 
and I.C. Reid, Chronic mild stress and sucrose consumption: validity as a model of depression, Physiol. Behav. 60 (1996), pp. 1481-1484.

Greenwood et al., 2003 B.N. Greenwood, T.E. Foley, H.E.W. Day, J. Campisi, S.H. Hammack, S. Campeau, S.F. Maier and M. Fleshner, Freewheel running prevents learned helplessness/behavioral depression: role of dorsal raphe serotonergic neurons, J. Neurosci. 23 (2003), pp. 2889 2898.

Harris et al., 1997 R.B. Harris, J. Zhou, B.D. Youngblood, G.N. Smagin and D.H. Ryan, Failure to change exploration or saccharin preference in rats exposed to chronic mild stress, Physiol. Behav. 63 (1997), pp. 91-100.

Harrison et al., 2001 A.A. Harrison, Y.T.B. Liem and A. Markou, Fluoxetine combined with a serotonin-1A receptor antagonist reversed reward deficits observed during nicotine and amphetamine withdrawal in rats, Neuropsychopharmacology 25 (2001), pp. 55-71.

Hatcher et al., 1997 J.P. Hatcher, D.J. Bell, T.J. Reed and J.J. Hagan, Chronic mild stress-induced reductions in saccharin intake depend upon feeding status, J. Psychopharmacol. 11 (1997), pp. 331-338.

Hodos, 1961 W. Hodos, Progressive ratio as a measure of reward strength, Science 134 (1961), pp. 933-934.

Hughes, 1965 R.N. Hughes, Food deprivation and locomotor exploration in the white rat, Anim. Behav. 13 (1965), pp. $30-32$.

Leith and Barrett, 1976 N.J. Leith and R.J. Barrett, Amphetamine and the reward system: evidence for tolerance and post-drug depression, Psychopharmacologia 46 (1976), pp. 19-25.

Lett et al., 2000 B.T. Lett, V.L. Grant, M.J. Byrne and M.T. Koh, Pairings of a distinctive chamber with the aftereffect of wheel running produce conditioned place preference, Appetite 34 (2000), pp. 87-94.

Maier and Seligman, 1976 S.F. Maier and M.E.P. Seligman, Learned helplessness: theory and evidence, J. Exp. Psychol. 105 (1976), pp. 3-46.

Markou and Koob, 1992 A. Markou and G.F. Koob, Construct validity of a self-stimulation threshold paradigm: effects of reward and performance manipulations, Physiol. Behav. 51 (1992), pp. 111-119.

McFarland and Ettenberg, 1999 K. McFarland and A. Ettenberg, Haloperidol does not attenuate conditioned place preferences or locomotor activation produced by food- or heroin-predictive discriminative cues, Pharmacol. Biochem. Behav. 62 (1999), pp. 631-641.
Olds and Milner, 1954 J. Olds and P. Milner, Positive reinforcement produced by electrical stimulation of septal area and other regions of the brain, J. Comp. Physiol. Psychol. 47 (1954), pp. 419-427.

Palmatier et al., 2003 M.I. Palmatier, E.Y.K. Fung and R.A. Bevins, Effects of chronic caffeine preexposure on the conditioned and unconditioned psychomotor activity induced by nicotine and amphetamine in rats, Behav. Pharmacol. 14 (2003), pp. 191-198.

Panksepp and Burgdorf, 2003 J. Panksepp and J. Burgdorf, "Laughing" rats as the evolutionary antecedents of human joy?, Physiol. Behav. 79 (2003), pp. 533-547.

Panksepp et al., 2004 J. Panksepp, C. Nocjar, J. Burgdorf, J.B. Panksepp and R. Huber, The role of emotional systems in addiction: a neuroethological perspective. In: R.A. Bevins and M.T. Bardo, Editors, Nebraska Symposium on Motivation, 50, Motivational Factors in the Etiology of Drug Abuse, University of Nebraska Press, Lincoln, NE (2004), pp. 85-126.

Papp, 1989 M. Papp, Differential effects of short- and long-term antidepressant treatments on the food-induced place preference conditioning in rats, Behav. Pharmacol. 1 (1989), pp. 69-74.

Papp et al., 1991 M. Papp, P. Willner and R. Muscat, An animal model of anhedonia: attenuation of sucrose consumption and place preference conditioning by chronic unpredictable mild stress, Psychopharmacology 104 (1991), pp. 255-259.

Pierce et al., 1990 R.C. Pierce, C.A. Crawford, A.J. Nonneman, B.A. Mattingly and M.T. Bardo, Effects of forebrain dopamine depletion on novelty-induced place preference behavior in rats, Pharmacol. Biochem. Behav. 36 (1990), pp. 321-325.

Sheldon, 1969 A.B. Sheldon, Preference for familiar versus novel stimuli as a function of the familiarity of the environment, J. Comp. Physiol. Psychol. 67 (1969), pp. 516521.

Valverde et al., 1997 O. Valverde, C. Smadja, B.P. Roques and R. Maldonado, The attenuation of morphine-conditioned place preference following chronic mild stress is reversed by a CCKB receptor antagonist, Psychopharmacology 131 (1997), pp. 79-85.

Willner, 1997 P. Willner, Validity, reliability and utility of the chronic mild stress model of depression: a 10-year review and evaluation, Psychopharmacology 134 (1997), pp. 319-329. 